\title{
Associations between sedentary time and EQ-5D index score in middle-aged and older adults.
}

\author{
Sang-Dol Kim* \\ Department of Nursing, College of Health Science, Kangwon National University, 346 Hwangjo-gil, Dogye-eup, \\ Samcheok-si, Gangwon-do, Republic of Korea
}

\begin{abstract}
Purpose: This study was conducted to identify the associations between sedentary time and EuroQol-5dimensional (EQ-5D) index score in middle-aged and older adults.

Materials and methods: Data were extracted from the 6th Korea National Health and Nutrition Examination Survey (2014-2015). Subjects aged $\geq 45$ y were selected for analysis $(n=3,543)$. Selfreported sedentary time and EQ-5D index score were the outcome variables. The sedentary time was dichotomized into $\geq 7.5 \mathrm{~h} / \mathrm{d}$ and $<7.5 \mathrm{~h} / \mathrm{d}$. To ensure that the sample represents the Korean population, sampling weights were generated by considering a complex sample. Associations with sedentary time and EQ-5D index score were analysed using multiple logistic regression.

Results: Sitting for $\geq 7.5 \mathrm{~h} / \mathrm{d}$ was significantly associated with $85 \%$ (odds ratio, 0.15 ; $95 \%$ confidence interval, 0.08-0.30) lower odds of EQ-5D index score when adjusted for sex and age compared to those who were sitting for $<7.5 \mathrm{~h} / \mathrm{d}$.

Conclusion: These findings imply that in order to improve health-related quality of life in middle-aged and older adults, sedentary time should be reduced.
\end{abstract}

Keywords: Sedentary time, EQ-5D index.

Accepted on April 13, 2018

\section{Introduction}

All humans experience aging throughout their life cycle. A successful aging process is an especially important task for middle-aged and older adults, which may be met through a high quality of life. The quality of life is an important concept and health indicator set by the World Health Organization [1]. However, previous studies suggested that one's health-related quality of life can negatively be affected by a sedentary behaviour in young adult men [2], adults with visual impairments [3], and adults [4,5]. Sedentary behaviour is defined as a sitting or reclining posture in a waking state [6] Prolonged sedentary time was reported as an important risk factor for poor health in middle-aged and older adults [7] and an independent predictor of adverse health outcomes in older adults [8]. Sedentary behaviour may also influence the overall success on the aging process physically, psychologically, and socially in middle-aged and older adults [7]. Taken together, sedentary time could be an important factor for successful aging and quality of life in middle-aged and older adults. Unfortunately, aside from the negative influences on the successful aging [7], the relationship between sedentary time and quality of life in middle-aged and older adults remains minimally explored $[9,10]$. In this context, the association of sedentary time with quality of life in middle-aged and older adults should be investigated. Therefore, the present study aimed to investigate the association between sedentary time and quality of life in a representative sample of middle-aged and older adults in Korea.

\section{Materials and Methods}

The subjects were selected from the 3,543 populations aged $\geq$ $45 \mathrm{y}$ in the $6^{\text {th }}$ Korea National Health and Nutrition Examination Survey (KNHNES, 2014-2015), which was approved by the institutional review board of the Korea Centers for Disease Control and Prevention (2013-07CON-03-4C and 2013-12EXP-03-5C) [11]. The main outcomes are sedentary time and EuroQol-5-dimensional (EQ-5D) index score. The sedentary time self-reported through interviews was dichotomized into sitting $\geq 7.5 \mathrm{~h} / \mathrm{d}$ and $<7.5 \mathrm{~h} / \mathrm{d}$ $[12,13]$. This cut-off time was based on a meta-analysis indicating that "the risk of all-cause mortality increases when a person self-reported to sit approximately $>7-8 \mathrm{~h} / \mathrm{d}$ " $[12,13]$. EQ-5D index scores are measured using a scale where 0 represents the "worst health state" and 1 represents a "perfect health state." Statistical analyses were conducted using the SPSS program, version 18.0 (IBM Corp., Armonk, NY) for Windows. To ensure that the sample represents the Korean population, sampling weights were generated by considering a complex sample. Associations between sedentary time and EQ-5D index score were analysed using multiple logistic regression. Probability values of $<0.05$ were considered statistically significant. 


\section{Results}

Tables 1 and 2 present the findings. The total number of subjects aged $\geq 45 \mathrm{y}$ was 3,543 with an average age of $59.37 \pm$ 0.3 (mean \pm standard deviation). Sitting for $\geq 7.5 \mathrm{~h} / \mathrm{d}$ was significantly associated with $85 \%$ (Odds Ratio (OR), 0.15 ; 95\% Confidence Interval (CI), 0.08-0.30) lower odds of the EQ-5D index score when adjusted for sex and age compared to those who were sitting for $<7.5 \mathrm{~h} / \mathrm{d}$. Sitting for $\geq 7.5 \mathrm{~h} / \mathrm{d}$ was significantly associated with $23 \%$ (OR, 0.77; CI, 0.65-0.92) lower odds of the EQ-5D index score in women than men. Compared to subjects aged 75 and older (reference group), sitting for $\geq 7.5 \mathrm{~h} / \mathrm{d}$ was significantly associated with $36 \%$ (OR, 0.64; CI, 0.48-0.86) and 39\% (OR, 0.61; CI, 0.46-0.81) lower odds of EQ-5D index score in subjects aged 55-64 y and 65-74 y, respectively.

Table 1. Characteristics of subjects aged $\geq 45(N=3,543)$.

\begin{tabular}{llll}
\hline Characteristics & \multicolumn{2}{l}{ Mean or \% (SE) } & \\
\cline { 2 - 4 } & Total & Male & Female \\
\hline Age, mean & $59.37(0.30)$ & $58.58(0.3)$ & $60.27(0.4)$ \\
\hline EQ-5D index, mean & $0.92(0.0)$ & $0.95(0.0)$ & $0.90(0.0)$ \\
\hline Sedentary time, mean & $7.09(0.12)$ & $7.32(0.17)$ & $6.84(0.11)$ \\
\hline$<7.5 \mathrm{~h} / \mathrm{d}$ & $51.4(1.4)$ & $49.7(1.9)$ & $52.9(1.5)$ \\
\hline$\geq 7.5 \mathrm{~h} / \mathrm{d}$ & $48.6(1.4)$ & $50.3(1.9)$ & $47.1(1.5)$ \\
\hline
\end{tabular}

SE: Standard Error.

Table 2. Associations between sedentary time and EQ-5D index among subjects aged $\geq 45 y$.

\begin{tabular}{llll}
\hline Characteristics & \multicolumn{3}{l}{ Adjusted for sex and age } \\
\cline { 2 - 4 } & \multicolumn{2}{l}{ Sedentary time $\geq \mathbf{7 . 5} \mathbf{~ h / d ~}$} & \\
\cline { 2 - 4 } & Weighted OR & $\mathbf{9 5 \%} \mathbf{C l}$ & p-value \\
\hline EQ-5D index & 0.15 & $0.08-0.30$ & 0 \\
\hline Sex & & & \\
\hline Female & 0.77 & $0.65-0.92$ & 0.003 \\
\hline Male & 1 & Reference & \\
\hline Age & & & 0.001 \\
\hline $45-54$ & 0.84 & $0.63-1.12$ & 0.242 \\
\hline $55-64$ & 0.64 & $0.48-0.86$ & 0.003 \\
\hline $65-74$ & 0.61 & $0.46-0.81$ & 0.001 \\
\hline $75+$ & 1 & Reference & \\
\hline
\end{tabular}

\section{Discussion}

In this study's results, the EQ-5D index scores in subjects sitting for 7.5 or more hours/day were significantly $(85 \%)$ lower than those sitting for less than $7.5 \mathrm{~h} / \mathrm{d}$ after adjusting for sex and age in the middle-aged and older adults. These findings are similar to those of a previous study in Australia, indicating that "individuals with the most extreme sedentary profiles may be vulnerable to additional losses of quality of life if they become more sedentary" in middle-aged and older adults with osteoarthritis [14]. Similarly, another previous study suggested that "restricted sedentary time has been demonstrated to positively influence health-related quality of life in adults without disabilities" in adults with visual impairments [3]. The present study findings support the results of previous studies that prolonged sedentary time is significantly associated with quality of life in adults with visual impairments or general adults or in middle-aged and older adults with osteoarthritis $[3,5,14]$. In contrast with previous studies indicating that men have lower quality of life than women, the present study show that the quality of life was lower in women than men when sitting for 7.5 or more $\mathrm{h} / \mathrm{d}$ in older adults or among older men $[9,10]$. These results suggest that a comparative study is needed by selecting different subjects. With regard to age, the quality of life in subjects aged 55-74 y was lower than those aged $\geq 75$ y who (reference group) were sitting for 7.5 or more hours/day. These results indicate that strategies for reducing sedentary time spent are needed in those who aged 55-74 y. Present study supported that reducing sedentary time spent may be useful strategies to improve health-related quality of life [8]. In conclusion, these findings imply that sedentary time should be reduced to improve the quality of life of middle-aged and older adults. In particular, appropriate interventions should be implemented to improve the quality of life and reduce the sedentary time in women aged 55-74 y.

\section{Acknowledgements}

Data in this study were extracted from the 6th Korea National Health and Nutrition Examination Survey (2015) conducted by the Korea Centers for Disease Control and Prevention.

\section{References}

1. The WHOQOL Group. The World Health Organization quality of life assessment (WHOQOL): position paper from the World Health Organization. Soc Sci Med 1995; 41: 1403-1409.

2. Paivarinne V, Kautiainen H, Heinonen A, Kiviranta I. Relations between subdomains of physical activity, sedentary lifestyle, and quality of life in young adult men. Scand J Med Sci Sports 2017.

3. Haegele JA, Famelia R, Lee J. Health-related quality of life, physical activity, and sedentary behavior of adults with visual impairments. Disabil Rehabil 2017; 39: 2269-2276.

4. Kim J, Im JS, Choi, YH. Objectively measured sedentary behavior and moderate-to-vigorous physical activity on the health-related quality of life in US adults: The National Health and Nutrition Examination Survey 2003-2006. Qual Life Res 2017; 26: 1315-1326.

5. Kolt GS, George ES, Rebar AL, Duncan MJ, Vandelanotte C, Caperchione CM, Maeder AJ, Tague R, 
Savage TN, Van Itallie A, Mawella NR, Hsu WW, Mummery WK, Rosenkranz RR. Associations between quality of life and duration and frequency of physical activity and sedentary behaviour: Baseline findings from the WALK 2.0 randomised controlled trial. PLoS One 2017; 12: 0180072.

6. Sedentary Behavior Research Network. Sedentary behavior research network. Sed Behav Netwrork 2012.

7. Shilpa D, Liza S. Sedentary behavior and physical activity are independent predictors of successful aging in middleaged and older adults. J Aging Res 2012; 2012.

8. Dogra S, Stathokostas L. Correlates of extended sitting time in older adults: an exploratory cross-sectional analysis of the Canadian community health survey healthy aging cycle. Int J Public Health 2014; 59: 983-991.

9. Meneguci J, Sasaki JE, Santos A, Scatena LM, Damiao R. Sitting time and quality of life in older adults: a population-based study. J Phys Act Health 2015; 12: 1513-1519.

10. Vallance Jeff K, Eurich DM, Alison LL, Celeste MJ, Steven T. Associations between sitting time and healthrelated quality of life among older men. Ment Health Phys Act 2013; 6: 49-54.

11. Korea Centers for Disease Control and Prevention. The 6th Korea National Health and Nutrition Examination Survey 2015.
12. Chau JY, Grunseit AC, Chey T, Stamatakis E, Brown WJ, Matthews CE, Bauman AE, van der Ploeg HP. Daily sitting time and all-cause mortality: a meta-analysis. PLoS One 2013; 8: 80000.

13. Lakerveld J, Loyen A, Schotman N, Peeters CFW, Cardon G, van der Ploeg HP, Lien N, Chastin S, Brug J. Sitting too much: A hierarchy of socio-demographic correlates. Prev Med 2017; 101: 77-83.

14. Pinto D, Song J, Lee J, Chang RW, Semanik PA, EhrlichJones LS, Pellegrini CA, Dunlop DD. Association between sedentary time and quality of life from the osteoarthritis Initiative: who might benefit most from treatment? Arch Phys Med Rehabil 2017; 98: 2485-2490.

\section{*Correspondence to}

Sang-Dol Kim

Department of Nursing

College of Health Science

Kangwon National University

Republic of Korea 